\title{
Polymeric microporous nanofilms as smart platforms for in vitro assessment of nanoparticle translocation and Caco-2 cell culture
}

\author{
Leonardo Ricotti, Member, IEEE, Giulia Gori, Daniele Cei, Joana Costa, Giovanni Signore, and Arti \\ Ahluwalia
}

\begin{abstract}
The study of nanomaterial translocation across epithelial barriers is often hindered by the low permeability of transwell membranes to nanoparticles. To address this issue ultra-thin poly(L-lactic acid) nanofilms with zero tortuosity micropores were developed for use in nanoparticle passage tests. In this study we demonstrate that microporous polymeric nanofilms allow a significantly higher passage of silver nanoparticles in comparison with commercial membranes normally used in Transwell inserts. A robust procedure for collecting free-standing nanofilms which enables their manipulation and use in lab-on-chip systems is described. We also demonstrate the cytocompatibility of porous nanofilms and their ability to sustain the adhesion and proliferation of Caco-2 cells. Ultra-thin microporous membranes show promise as lowcost nanomaterial screening tools and may be used as matrices for the development of bioengineered systems for mimicking the intestinal epithelium.
\end{abstract}

Index Terms - Caco-2 cells, Lab-on-chip, Nanoparticle translocation, Passage tests, Polymeric nanofilms, Porous membranes, Ultra-thin films, Zero tortuosity micropores.

\section{INTRODUCTION}

$\mathrm{T}$ HE impact of nanomaterials on human tissues/organs is still under debate, particularly as regards long term and chronic effects [1,2]. In this context, the ability of small sized particles to cross the intestinal barrier represents a major

This paper was submitted for review on July 1, 2016

This work was supported in part by the FUTURA project (Focused Ultrasound Therapy Using Robotic Approaches, http://www.futuraproject.eu/, funded in the FP7 EU framework. Grant agreement no. 611963) and by the M2Neural project (http://www.m2neural.eu), funded in the FP7 M-ERA.NET Transnational framework. The study was also partly funded by the Italian Ministry of Education, University \& Research (MIUR) (Project PRIN 2010, MIND, 2010J8RYS7), while J. Costa is recipient of a EU H20202 Marie Curie ITN Grant (MICACT).

L. Ricotti is with the BioRobotics Institute of Scuola Superiore Sant'Anna, Pontedera (Pisa), 56025 Italy (e-mail: 1.ricotti@sssup.it).

G. Gori was with the BioRobotics Institute of Scuola Superiore Sant'Anna, Pontedera (Pisa), 56025 Italy and with the Research Center "E.Piaggio", University of Pisa, Pisa, 56126 Italy (e-mail: giulia.g2608@ hotmail.it)

D. Cei and J. Costa are with the Research Center "E.Piaggio", University of Pisa, Pisa, 56126 Italy and with IVTech S.r.l., Massarosa (Lucca), 55054 Italy (e-mails: daniele.cei87@gmail.com and j.costa@ivtech.it).

G. Signore is with the IIT@NEST-Center for Nanotechnology Innovation, Pisa, 56127 Italy (e-mail: giovanni.signore@iit.it).

Arti Ahluwalia is with the Research Center "E.Piaggio", University of Pisa, Pisa, 56126 Italy (e-mail: arti.ahluwalia@unipi.it). health concern that is attracting growing attention [3], due to the critical role of intestinal epithelial cells (IECs). In fact, alterations in IEC function are thought to contribute to the emergence of several pathological conditions, such as inflammatory bowel disease, type I diabetes, rheumatoid arthritis and multiple sclerosis [4].

In vivo studies have been conducted to assess the distribution of nanoparticles in whole organisms and their effects due to accumulation in specific organs/tissues. However, it would be highly desirable to reduce animal studies as much as possible for ethical and economic reasons. In addition, animal models do not reliably simulate human physiology [5]. Thus, advanced in vitro models are needed to study nanoparticle translocation and to estimate in vivo translocation and exposure.

The gold standard to investigate in vitro the behavior of IECs and, in general, of the human intestinal barrier is the immortalized cell line, Caco-2. Monolayers of Caco-2 serve as models of enterocytes, the most abundant epithelial cell type in the intestine, and they have been extensively used to predict the translocation of nanomaterials through the human gut [6$10]$.

The models can be refined by co-culturing Caco- 2 cells with other cell types, such as mucus-secreting HT29-MTX human colon carcinoma cells (used to enrich the in vitro microenvironment with a mucus layer consisting of mucin glycoproteins) and microfold (M) cells, used to better replicate the complex anatomy of the gut epithelium and the ability to uptake and translocate relatively large particles [11].

In all the above-mentioned models, a permeable membrane supporting the cell culture or co-culture is needed. Such membrane should offer zero resistance to the passage of materials so as not to interfere with cell-mediated nanoparticle passage. To minimize nanoparticle agglomeration and resistance to passage, the ideal membrane should have a thickness close to the characteristic dimensions of the nanoparticles and zero tortuosity. Most in vitro barrier models rely on cells cultured on Transwell inserts, composed of a polyester or polycarbonate permeable membrane that separates, through the cell layer, an apical and a basolateral compartment. Depending on the cell type selected, the Transwell model can be used to investigate nanoparticle translocation in the lungs, in the skin, in the placenta or in the 
gut. Transwell inserts have pore sizes ranging from 0.4 to 8 $\mu \mathrm{m}$, an overall porosity of $\sim 20 \%$ and thickness values ranging from 10 to $50 \mu \mathrm{m}$. Such values are a few orders of magnitude larger than the typical size of potentially hazardous nanoparticles that can enter the body, such as carbon black (14 - $66 \mathrm{~nm})$ [12], titanium dioxide $(20-200 \mathrm{~nm})$ [13], silver nanoparticles $(5-100 \mathrm{~nm})$ [14] and carbon nanotubes (5 $100 \mathrm{~nm}$ in diameter, $50 \mathrm{~nm}$ - few $\mu \mathrm{m}$ in length) [15], just to mention few examples. Of particular concern in this context, are metal and metal oxide nanoparticles such as silver, titanium dioxide, gold and iron, which have a tendency to cluster and agglomerate, particularly at high concentrations [16]. These nanoparticles can remain entrapped within the 3D, often tortuous, pores of the membrane, giving rise to false negatives [17].

Some attempts to engineer ultra-thin porous membranes with straight through zero tortuosity pores (that do not deviate fluid streamlines) have been reported in the literature. Rosembloom and colleagues developed a silicon carbide nanoporous membrane obtained by electrochemical etching [18]. This device allowed the diffusion of proteins up to $29,000 \mathrm{Da}$, while excluding larger ones. Such a system is unsuitable for nanoparticle translocation tests in an intestinelike barrier, since nanoparticles can cover a broad range of dimensions. Other groups reported the use of microporous membranes for lab-on-chip applications [18-21]. However, these systems are rather rigid and excessively costly, or too thick to prevent nanoparticles clogging in the pores.

Polymeric nanofilms are quasi-two-dimensional (2-D) structures, characterised by a very small thickness (from tens to hundreds of nanometers) and a very large surface area (up to several square centimeters). Poly(L-lactic acid) (PLLA) has been demonstrated to be particularly suitable for the development of robust, yet highly flexible free-standing nanomembranes featured by optical transparency, low-cost and ease of fabrication (based on spin-assisted deposition) [22]. The potential of PLLA nanofilms for a series of biomedical applications has been recently highlighted, ranging from surgical sealing $[23,24]$ to regenerative medicine [25] and drug delivery [26]. However,- microporous PLLA nanofilms and their application in lab-on-chip systems have not been reported.

In this paper we describe a fabrication procedure to obtain PLLA ultra-thin films with regularly distributed micropores. We performed nanoparticle passage tests by comparing the permeability over time of the microporous nanofilms with those of commercial membranes, normally used in Transwell inserts. Finally, Caco-2 cells were cultured on the nanofilms to verify their biocompatibility.

\section{MATERIALS AND MethodS}

\section{A. Nanofilm fabrication}

The fabrication procedure used to obtain free-standing microporous nanofilms is schematised in Fig. 1. Each step of the process was carried out in a clean-room (class 1000) to avoid contamination. A positive photoresist-based mold
(Shipley S1813) provided with micropillars (pillar diameter: $4.94 \pm 0.43 \mu \mathrm{m}$, pillar height: $1.50 \pm 0.10 \mu \mathrm{m}$ ) was developed through lithographic techniques and a dedicated photomask The photolithography set-up included a wet bench (Arias $\mathrm{GmbH}$ ) and a mask aligner (Suss Microtech $\mathrm{GmbH}$, MA6) with a $350 \mathrm{~W}$ Hg lamp. Molds were gold sputtered ( $25 \mathrm{~mA}, 20$ s - Quorum Technologies, Q150R ES): the inert gold coating prevented the PLLA solution from chemically reacting with the underlying photoresist layer. Then, they were plasma treated $\left(30 \mathrm{~W}, 90 \mathrm{~s}, 50 \% \mathrm{O}_{2}\right.$ - Gambetti SpA, Colibrì). A sacrificial layer of polyvinyl alcohol (PVA) and a PLLA layer were then deposited onto the mold by spin-assisted deposition (WS-650 spin processor, Laurell Technologies Corp., North Wales, PA). PVA $\left(1 \% \mathrm{w} / \mathrm{v}\right.$ in deionised water or $\left.\mathrm{d}-\mathrm{H}_{2} \mathrm{O}\right)$ was spun at 4,000 rpm for $20 \mathrm{~s}$. PLLA $(2 \% \mathrm{w} / \mathrm{v}$ in dichloromethane) was spun for $20 \mathrm{~s}$, at different speeds, ranging from 3,000 to $7,000 \mathrm{rpm}$. After spinning, a thermal treatment $\left(80{ }^{\circ} \mathrm{C}\right.$ for $\left.60 \mathrm{~s}\right)$ was used to dry the system and to obtain a robust and uniform PLLA membrane. Finally, after immersion in $\mathrm{d}-\mathrm{H}_{2} \mathrm{O}$, the PVA layer was dissolved and the PLLA membrane released.

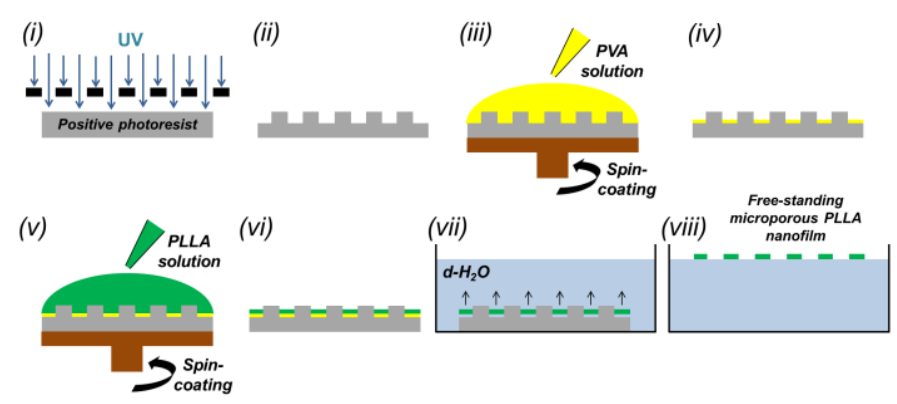

Fig. 1. Scheme of the porous nanofilm fabrication steps: micropillar-based mold development (i, ii), deposition of sacrificial layer (iii, iv), deposition of PLLA layer (v, vi) and release of the porous nanofilm in water (vii, viii).

\section{B. Nanofilm characterization}

The porous nanomembranes were imaged by scanning electron microscopy (SEM) using an EVO MA15 instrument (Zeiss) equipped with LaB6 source and working at a $10 \mathrm{kV}$ accelerating voltage. Nanofilm thickness was evaluated by means of a stylus profilometer (KLA-Tencor, P-6).

Nanofilm mechanical properties were measured using the strain-induced elastic buckling instability for mechanical measurement (SIEBIMM) technique [27]. The SIEBIMM test enables calculation of the elastic modulus by measuring the buckling wavelength of the nanomembrane collected on a mechanically pre-stretched slab of PDMS. The nanofilm elastic modulus (E) was calculated as follows:

$$
E=\frac{3 \cdot E_{P D M S} \cdot\left(1-v_{P L L A}{ }^{2}\right)}{\left(1-v_{P D M S}{ }^{2}\right)} \cdot\left(\frac{\lambda}{2 \pi \cdot x}\right)^{3}
$$

where EPDMS is the PDMS elastic modulus (1.80 MPa), $v_{\text {PLLA }}$ and $v_{\text {PDMS }}$ are the Poisson's moduli of PLLA nanofilm (0.33) and of PDMS (0.5), respectively, $\mathrm{x}$ is the initial PDMS slab length $(4 \mathrm{~cm})$ and $\lambda$ is the wavelength of the buckling pattern measured using a microscope. 


\section{Nanomaterials}

Different nanoparticles (NPs) with different agglomeration properties were investigated. PS-FITC (PolystyreneFluorescein isothiocyanate) Fluoresbrite ${ }^{\circledR}$ NPs (Polyscience Inc., Germany) have been shown to remain well dispersed even at high concentrations [28]. PS-FITC NPs featured by two different diameters were investigated: $211 \mathrm{~nm}$ and $55 \mathrm{~nm}$, respectively. On the other hand, silver NPs (Ag NPs, NM300 from Ras $\mathrm{GmbH}$, an OECD) with a nominal diameter of 20 $\mathrm{nm}$, which tend to form clusters even at low concentrations, were used as a model of a typical metal/metal oxide engineered nanoparticle. The NPs were prepared and used following the protocol described in Ucciferri et al. [28]. All NPs were characterized in the culture medium described in section II.B. The PS-FITC NPs with a nominal diameter of 55 $\mathrm{nm}$ showed a hydrodynamic diameter in culture medium of 55 $\pm 4 \mathrm{~nm}$ and a monomodal size distribution with poly-dispersity index of $0.02 \pm 0.00$, while the $211 \mathrm{~nm}$ PS-FITC NPs showed a size of $419 \pm 23 \mathrm{~nm}$ with a dispersity index of $0.14 \pm 0.03$. $\mathrm{Ag}$ NPs showed a nominal diameter of $20 \mathrm{~nm}$ and a hydrodynamic diameter in medium of $120 \pm 4 \mathrm{~nm}$. In addition, FITC (Sigma-Aldrich) dissolved in culture medium was employed as a non-particle (soluble) control.

\section{Translocation tests}

Translocation (passage) tests were carried out on PLLA microporous nanofilms, by using the above-mentioned nanomaterials and compound and by comparing the results with those obtained with standard commercial polycarbonate membranes (ISOPORE ${ }^{\circledR}$ ), normally used in Transwell inserts. The tests were carried out using the set-up shown in Fig. 2: after $10 \mathrm{~min}$ sonication, $500 \mu \mathrm{L}$ of solution containing the target compound or nanoparticle at a known concentration in Dulbecco's Modified Eagle Medium (DMEM) supplemented with $10 \%$ fetal bovine serum (FBS) were pipetted onto the membrane (PLLA nanofilm or polycarbonate membrane), mounted and secured in a teflon holder. At different timepoints (5 min, $10 \mathrm{~min}, 30 \mathrm{~min}, 1 \mathrm{~h}, 2 \mathrm{~h}, 4 \mathrm{~h}, 6 \mathrm{~h}, 8 \mathrm{~h}$ and $24 \mathrm{~h}$ ), $100 \mu \mathrm{L}$ of solution were sampled from the basolateral compartment (below the membrane) assayed using a fluorimeter or inductive coupled plasma mass spectroscopy (ICP-MS) measurements. All measurements were performed on four independent samples for each time-point.

Fluorescence measurements allowed quantification of the amount of FITC and PS-FITC NPs. FITC is featured by a peak excitation wavelength of $495 \mathrm{~nm}$ and a peak emission wavelength of $525 \mathrm{~nm}$. It was added to the apical compartment of the system at a concentration of $40 \mu \mathrm{g} / \mathrm{mL}$. The two PSFITC NP types were both featured by a peak excitation wavelength of $441 \mathrm{~nm}$ and a peak emission wavelength of 486 $\mathrm{nm}$. They were added to the apical compartment of the system at a concentration of $1 \mathrm{mg} / \mathrm{mL}$. Fluorescence measurements were carried out by using a plate reader (Perkin Elmer, VICTOR X3) provided with proper optical filters. Calibration curves were used to quantify compound and nanoparticle concentrations. All measurements (for each sample type and for each time-point) were performed in triplicate.

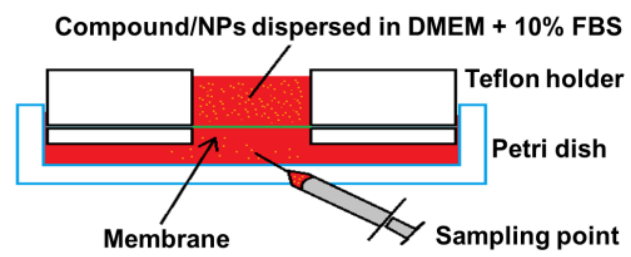

Fig. 2. Scheme of the set-up and procedure used for translocation tests.

ICP-MS measurements allowed quantification of Ag NPs. For each time-point, the collected solutions were extensively sonicated and $2 \mu \mathrm{L}$ were dissolved in $200 \mu \mathrm{L}$ of nitric acid for trace analysis. The solution was then digested in a microwave reactor (Discover SP-D, CEM) for $20 \mathrm{~min}$ at $200{ }^{\circ} \mathrm{C}$. After digestion, the sample was diluted to $2 \mathrm{~mL}$ with water (ICP-MS grade) and analyzed by ICP-MS (Agilent Technologies 7700 Series ICP-MS). Silver content was determined by comparison with a standard curve. All measurements (for each sample type and for each time-point) were performed in triplicate.

Compound/nanoparticle passage was computed by considering the cumulative data derived from measurements at the basolateral compartment, for the different time-points. The passage was then reported as a percentage of the initial compound/nanoparticle amount that had been pipetted onto the membrane, at the apical compartment.

\section{E. Cell cultures and fluorescence images}

Caco-2 cells were cultured in high glucose DMEM supplemented with $1 \%$ non-essential amino acids and containing 10\% FBS, $4 \mathrm{mM}$ glutamine, 100 U.I./mL penicillin and $100 \mu \mathrm{g} / \mathrm{mL}$ streptomycin (all reagents from SigmaAldrich, Italy). Subculture was performed at $50 \%$ confluence and the experiment was performed with the cell passage 48 . Before cell seeding, the devices were sterilized by exposure to UV light on either side for $30 \mathrm{~min}$. Cells were seeded at a density of $3 \times 10^{5}$ cells $/ \mathrm{cm}^{2}$ and maintained in culture for 10 days, changing the medium every two or three days. The membranes were monitored with an optical microscope at regular intervals.

At the end of the experiments the cells were fixed with $4 \%$ paraformaldehyde (PFA) for $20 \mathrm{~min}$ at room temperature, permeabilized with $0.1 \%$ Triton X-100 in PBS and stained with DAPI (4' 6-diamidino-2-phenylindole; $1 \mu \mathrm{g} / \mathrm{mL}$ in $1 \%$ PBS) for $10 \mathrm{~min}$ and with rhodamine-labeled phalloidin (Invitrogen). The samples were imaged using a confocal microscope (A1 Confocal Microscope System, Nikon Italy).

\section{F. Statistical analyses}

The data collected were subjected to analysis of variance in order to evaluate statistically significant differences among samples. A t-test was performed for comparison between two groups, while Holm-Sidak tests were performed for comparisons among several groups. Significance was set at $5 \%$. 


\section{RESULTS}

\section{A. Nanofilm fabrication, imaging and thickness measurements}

SEM images confirmed the presence of a regular distribution of micropores with zero tortuosity, as shown in Fig. 3A. Previous studies have demonstrated that the thickness of ultra-thin polymeric films can be varied by simply regulating the spinning speed [22-26]. Fig. 3B shows how the thickness decreases polynomially with spinning speed trend and indicates that PLLA membranes with sub-micrometric thickness can be obtained simply by a reduction in spin rpm. Obviously, a reduction in thickness implies increased fragility of the system and more difficulty in membrane handling. We chose $6,000 \mathrm{rpm}$, a speed which results in membranes with a thickness of $440 \pm 27 \mathrm{~nm}$, which is good compromise between ultra-thinness and the ability to manipulate and assemble the system as described in the following section.

Because of the delicate nature of the membranes, a method for efficient and reliable nanomembrane manipulation and securing is crucial for their successful application [22]. For this reason, a dedicated holder was designed to collect the nanofilm from $\mathrm{d}-\mathrm{H}_{2} \mathrm{O}$ and to keep it in a steady position during the nanoparticle translocation and cell culture experiments. Fig. 3C shows a CAD model of the holder parts and the teflonbased prototypes, secured by nylon screws.

(A)
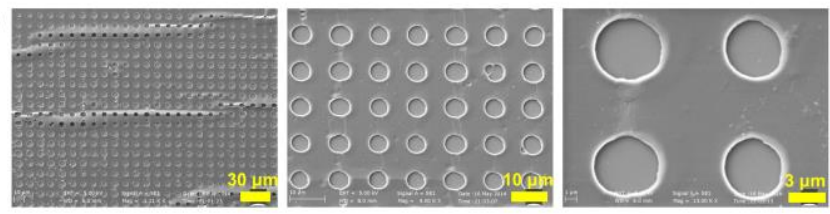

(B)

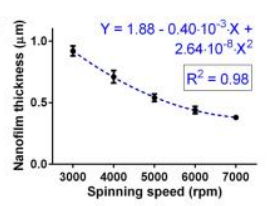

(C)
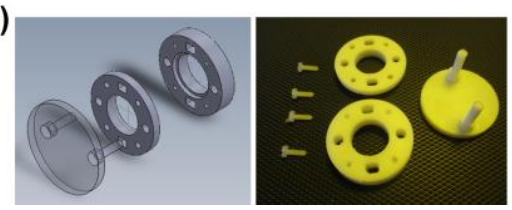

Fig. 3. (A) Scanning electron microscopy (SEM) images at different magnifications, showing the surface of microporous nanofilms. (B) Nanofilm thickness characterization for different spinning speeds. For each speed, 3 independent samples were tested and 3 different measurements were performed on each sample. (C) Computer assisted design (CAD) model of the holder and prototype components made of teflon, provided with nylon screws.

\section{B. Nanofilm mechanical properties}

Fig. 4 shows images of the stretched and clamped PDMS slab used for carrying out the SIEBIMM procedure (Fig. 4A) and of a PLLA nanofilm, buckled after PDMS strain relaxation (Fig. 4B). A continuous buckling pattern was clearly observed on the surface of the PLLA membrane and allowed estimation of the elastic modulus using Equation 1. The measured PLLA nanofilm elastic modulus was $2.64 \pm$ $0.37 \mathrm{GPa}$, which is significantly lower than the bulk elastic modulus of PLLA (7-10 GPa) [29]. Interestingly, previous reports on PLLA nanofilm mechanical properties, measured with the SIEBIMM technique, showed larger values (from 3.5 to $7 \mathrm{GPa}$ ) in comparison with the ones found in this study [30]. The smaller values found in our case can be ascribed to the presence of micropores, which confer higher distensibility to the membrane.
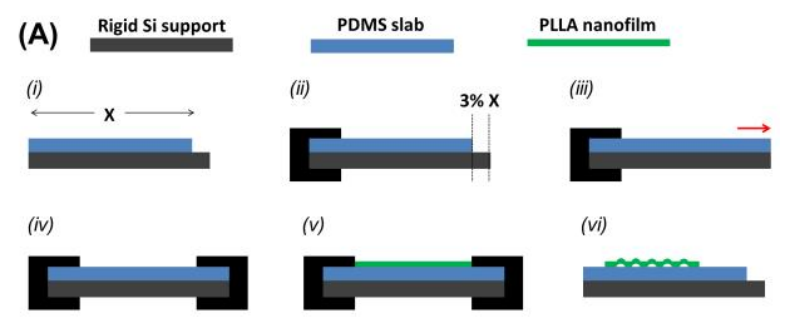

(vi)

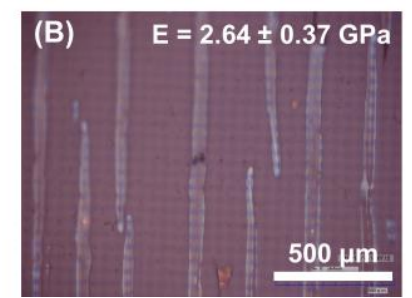

Fig. 4. Nanofilm mechanical properties measured using the SIEBIMM technique: (A) a PDMS slab is stretched onto a rigid supporting substrate and clamped (i-iv); then, the PLLA nanofilm is placed on the PDMS substrate (v) and the clamps are removed, thus triggering nanofilm buckling (vi). (B) Optical microscopy image showing a buckling pattern on the nanomembrane, from which the corresponding elastic modulus value can be calculated.

\section{Nanofilm manipulation}

Although it is seemingly straightforward, nanofilm recollection from water and its assembly within the holder are rather tricky procedures. Fig. 5 reports the different steps needed to properly manipulate the microporous nanofilms, with the aim of securing them safely and reliably on the holders.
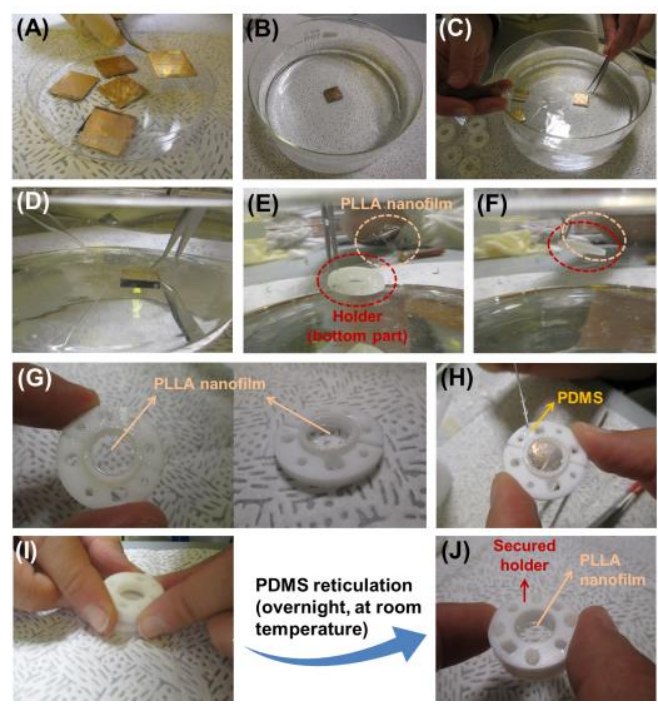

Fig. 5. Nanofilm collection and holder assembly. (A,B) PVA and PLLA are successively deposited on a mold with micropillar and immersed in $\mathrm{d}-\mathrm{H}_{2} \mathrm{O}$. (C,D) After dissolution of the PVA sacrificial layer, nanofilm detachment is facilitated by using tweezers and by gently directing a flow of water towards the edges of the nanofilm using a Pasteur pipette. (E-G) Once the film is completely detached from the mold, it is collected by approaching the base of the holder towards the nanofilm and then slowly pulling out the system from d- $\mathrm{H}_{2} \mathrm{O}$. $(\mathrm{H}, \mathrm{I})$ Non-reticulated PDMS solution is deposited on the external nanofilm area, then the holder top part is assembled and secured by nylon screws. (J) After reticulation, achieved by keeping the system overnight at room temperature, the PLLA microporous nanomembrane is stably secured within the holder. 
After releasing nanofilms from the mold in $\mathrm{d}-\mathrm{H}_{2} \mathrm{O}$ (Fig. 5A$\mathrm{D})$, the membranes were gently collected onto the bottom part of the holder (Fig. 5E-G) to which a thin film of PDMS monomer was applied around the external border (Fig. 5H). Once the monomer is cured, the nanomembrane is fixed to the holder and the remaining parts are gently screwed on to hold it snug. (Fig. 5I,J). The shape of the holder facilitates manipulation of the films and their insertion in standard 6-well plates. It also maintains a constant liquid volume below the membrane, thus reducing the risk of film rupture.

\section{Compound and nanoparticle translocation tests}

The results of translocation tests are shown in Fig. 6.
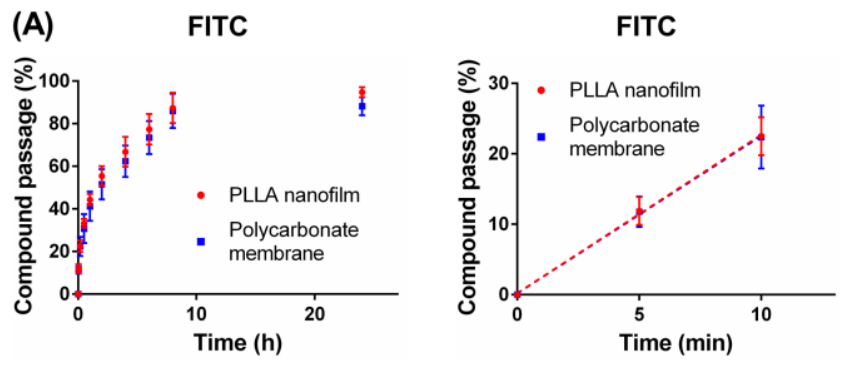

\section{(B) PS-FITC NPs - $200 \mathrm{~nm}$}
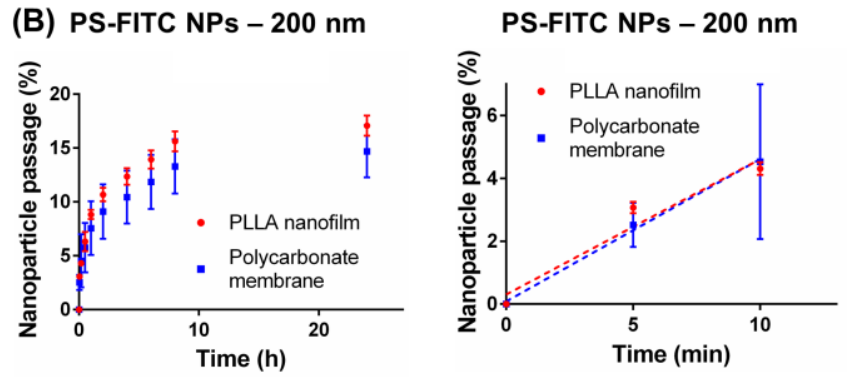

(C) PS-FITC NPs $-50 \mathrm{~nm}$
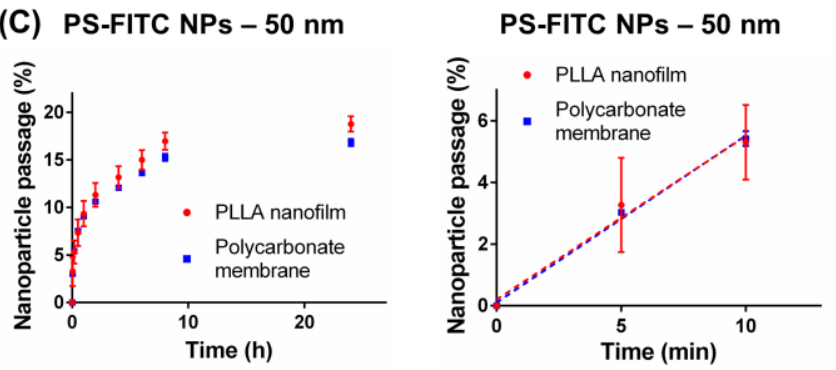

(D)

Ag NPs
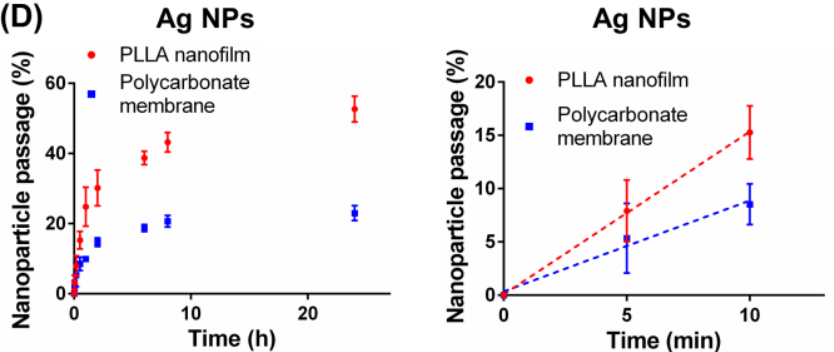

Fig. 6. Results of FITC and nanoparticle passage experiments for PLLA nanofilms and polycarbonate membranes. Graphs on the left report long-term passage (up to $24 \mathrm{~h}$ ), graphs on the right show details of short-term passage (up to $10 \mathrm{~min}$ ), for :(A) FITC ;(B) PS-FITC NPs - diameter: $200 \mathrm{~nm}$; (C) PSFITC NPs - diameter: $50 \mathrm{~nm}$; (D) Ag NPs. Compound/nanoparticle passage is reported as a percentage of the initial amount of compound/nanoparticle pipetted onto the membrane, at the apical compartment, before starting the experiment. Linear fits (right pictures) are based on data corresponding to three time-points: 0,5 and $10 \mathrm{~min}$.
FITC and nanoparticle passage was quantified for both PLLA nanofilms and standard polycarbonate membranes, for up to $24 \mathrm{~h}$ after starting the experiment. They were fitted using the following function [31]:

$y=a \cdot\left(1-b \cdot e^{-\left(t / t^{*}\right)}\right)$

where $a$ and $b$ are the fitting constants and $\mathrm{t}^{*}$ is the characteristic passage time.

Nanoparticle translocation is approximately linear in the first $10 \mathrm{~min}$. In this interval, the data were fitted to a straight line to calculate the apparent permeability of the membranes, using equation 3 [32]:

$P_{a p p}=\frac{V}{A} \cdot \frac{d C}{d t} \cdot \frac{1}{C_{0}}$

where $V$ is the volume of the basolateral compartment (4 $\mathrm{mL}), A$ is the membrane area exposed to the nanoparticle, $d C / d t$ is the rate of change of concentration in the basolateral compartment, calculated as the slope of the linear fitting (Fig. 6 , graphs in the right hand column). $C_{0}$ is the initial concentration in the apical compartment (above the membrane).

Table I reports the $\mathrm{t}^{*}$ and $\mathrm{P}_{\text {app }}$ values for the PLLA nanofilms and the polycarbonate membranes tested, for the different compound and nanoparticle types.

\begin{tabular}{|c|c|c|c|c|}
\hline \multirow[t]{2}{*}{$\begin{array}{l}\text { Compound/ } \\
\text { NP type }\end{array}$} & \multicolumn{2}{|c|}{ PLLA nanofilms } & \multicolumn{2}{|c|}{$\begin{array}{l}\text { Polycarbonate } \\
\text { membranes }\end{array}$} \\
\hline & $t^{*}(h)$ & $\mathrm{P}_{\text {app }}(\mathrm{cm} / \mathrm{s})$ & $\mathrm{t}^{*}(\mathrm{~h})$ & $\mathrm{P}_{\text {app }}(\mathrm{cm} / \mathrm{s})$ \\
\hline FITC & 2 & $\begin{array}{c}(11.30 \pm 0.02) \\
\times 10^{-3}\end{array}$ & 2 & $\begin{array}{c}(11.30 \pm 0.03) \\
\times 10^{-3}\end{array}$ \\
\hline $\begin{array}{c}\text { PS-FITC NPs } \\
-200 \mathrm{~nm}\end{array}$ & 2 & $\begin{array}{c}(1.96 \pm 0.07) \\
\times 10^{-3}\end{array}$ & 2 & $\begin{array}{c}(1.81 \pm 0.28) \\
\times 10^{-3}\end{array}$ \\
\hline $\begin{array}{c}\text { PS-FITC NPs } \\
-50 \mathrm{~nm}\end{array}$ & 2 & $\begin{array}{c}(2.11 \pm 0.20) \\
\times 10^{-3}\end{array}$ & 2 & $\begin{array}{c}(2.11 \pm 0.18) \\
\times 10^{-3}\end{array}$ \\
\hline $\mathrm{Ag}$ NPs & 3.5 & $\begin{array}{c}(3.80 \pm 0.10) \\
\times 10^{-3}(* *)\end{array}$ & 2 & $\begin{array}{c}(0.25 \pm 0.17) \\
\times 10^{-3}\end{array}$ \\
\hline
\end{tabular}

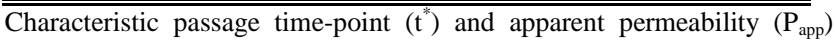
values for PLLA microporous nanofilms and polycarbonate membranes and for the different compound/nanoparticles tested. $* *=p<0.01$.

Results demonstrate that a slightly larger quantity of FITC and PS-FITC NPs of both sizes (200 $\mathrm{nm}$ and $50 \mathrm{~nm}$ ) passed through PLLA nanofilms than through the polycarbonate membranes after $24 \mathrm{~h}$, but the apparent permeability was the same for the two systems. A different behaviour was observed with Ag NPs. In this case, the apparent permeability was significantly $(\mathrm{p}<0.01)$ larger in PLLA nanofilms in comparison with the polycarbonate membranes. Furthermore, more than twice the amount of Ag NPs passed through the nanofilms at $24 \mathrm{~h}$ than the polycarbonate membranes. This implies a significantly higher plateau value and thus justifies the higher $\mathrm{t}^{*}$ value found for PLLA nanofilms, which could appear in contradiction to $\mathrm{P}_{\text {app }}$ values. 


\section{E. Caco-2 cell culture on the microporous nanomembranes}

In view of the possible application of PLLA microporous nanofilms as components of gut-mimicking bioengineered environments, we performed preliminary biological tests to evaluate the ability of nanomembranes to support intestinal epithelial cell cultures. Fig. 7A and Fig. 7B show that a homogeneous Caco-2 cell monolayer can be observed on the nanofilm surface after 10 days of culture. Fig. 7C is a SEM image at the same magnification of Fig. 7B, to compare micropore and cell distribution. The images show that the regularly distributed micropores with a diameter of $5 \mu \mathrm{m}$ do not hamper the formation of a homogeneous cell monolayer on the film surface.
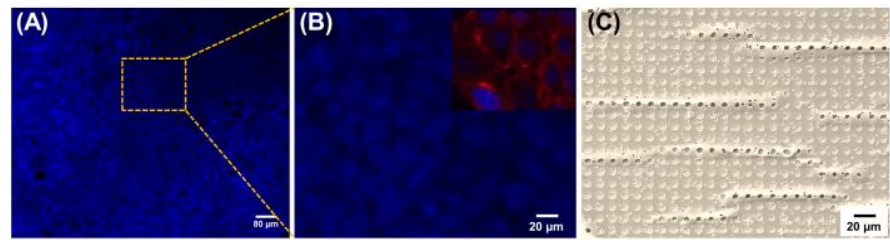

Fig. 7. Results of preliminary cell culture tests. Caco-2 cell nuclei are shown in blue in low-magnification (A) and high-magnification (B) fluorescence images. The inset shows Caco- 2 cell cytoskeleton (F-actin) in red and nuclei in blue. (C) SEM image of the porous nanofilm surface.

\section{DISCUSSION}

As engineered nanomaterials are increasingly used in food packaging materials, textiles, medicine and cosmetics, a better understanding of their potential hazard and impact on human health using non-animal methods is crucial for economic and ethical reasons [33]. One of the drawbacks of existing in vitro models (mostly based on standard Transwell inserts) used to study nanoparticle passage across biological barriers is the limited "transparency" of commercial membranes to nanoparticle passage, which adds a bias to the evaluation of nanoparticle translocation across cells. This is particularly true for engineered metal and metal oxide (e.g. $\mathrm{TiO}_{2}, \mathrm{Au}, \mathrm{Ag}, \mathrm{Fe}$ ) nanomaterials which agglomerate spontaneously and hence adhere to and clog the pores of Transwell membranes.

The results reported in section III.D demonstrate that PLLA microporous nanofilms are excellent alternatives to commercial membranes; besides their superior permeability, they are simple to fabricate and lower in cost. Moreover, the diameter and spacing of the nanofilm pores result in an overall porosity of $\sim 20 \%$, which is very similar to the nominal porosity of the polycarbonate membranes. The main differences in terms of short and long-term passage kinetics between the two systems, shown in Fig. 6, were found for Ag NPs. These metal NPs pass through the microporous nanofilms significantly faster and in greater amounts than polycarbonate membranes. Likely the sub-micrometric thickness of the ultra-thin membranes and zero tortuosity of the nanofilm pores minimise nanoparticle entrapment in the membrane matrix.

The results highlighted a significant difference between polycarbonate membranes and PLLA nanofilms, in terms of translocation efficacy, for Ag NPs, but not for PS ones. It could be argued that such difference depends on the different detection method used for the two nanoparticle types, namely spectrophotometric readings for PS NPs and ICP-MS measurements for $\mathrm{Ag}$ NPs. Although ICP-MS is more sensitive than spectrophotometric techniques, this does not appear as a crucial factor. In fact, for both PS and Ag NPs, the standard deviations obtained for the different measurements are comparable. Thus differences are due to the nature of the nanoparticles and two main mechanisms are probably involved. Firstly, as mentioned, Ag nanoparticles tend to form rather large and in some cases anisotropic aggregates [16]. This may imply a significantly higher entrapment rate in polycarbonate membranes, in comparison with PS NPs. Microporous nanofilms may reduce this entrapment rate thanks to their ultra-low thickness (comparable with the size of nanoparticles or nanoparticle aggregates) and zero tortuosity. Secondly, the nanoparticle charge may also play a role, but this appears as a less relevant factor. In fact, both PS and Ag NPs have a negative surface charge, while PLLA is slightly positive [34]. On the other hand, polycarbonate membranes are almost electrically neutral. Thus, it is unlikely that surface charge contributes to the improved performance of the microporous nanofilms, with the tested nanoparticle types. It is worth mentioning that nanofilm surface charge can be easily changed, by using the $\mathrm{D}(-)$ isomer of lactic acid, instead of the L(+) one [35]. Previous literature reports already demonstrated the possibility of fabricating poly(D-lactic acid) (PDLA) ultra-thin films, exploiting the same techniques used to fabricate PLLA ones [36]. Thus, different versions of the microporous nanofilms, based on PLLA or PDLA depending on the surface charge of the nanoparticle to be tested, can be envisioned.

Although several reports describe nanofilm fabrication and characterization [22-26, 37, 38], few studies describe detailed strategies to overcome some of the practical issues associated with manipulation and mounting. In some studies, nanofilm flexibility has been claimed as a feature allowing them to be cyclically aspirated and ejected through pipettes or syringes. Indeed, this would enable the collection and subsequent injection of drug- or cell-loaded nanofilms for drug delivery or regenerative medicine purposes $[25,26,37]$. The application of nanofilms during surgical and medical procedures may be facilitated by using thick supporting layers coupled with the membranes. Supporting sacrificial layers would allow manipulation and positioning of the nanofilm in the desired site, before dissolving in physiological media [23, 24, 39]. Finally, for some applications, nanofilms have been designed to be built directly onto the target device or tissue $[36,40]$. The above-mentioned strategies can be hardly applied in the field of lab-on-chip systems. Thus, the procedure described in section III.C (Fig. 5) represents a novelty in the state-of-the-art of polymeric nanomembranes for in vitro applications.

Although cell adhesion on microstructured polymers [41, 42] and PLLA nanofilm biocompatibility towards different cell types have been previously reported [25, 30, 36-38, 43], none of the studies use Caco-2 cells. In fact, despite being an immortalized cell line, they are considered difficult to culture reproducibly, particularly on non-standard substrates [44]. This is the first study describing the adhesion and sustained culture of Caco-2 cells on perforated PLLA nanomembranes and demonstrates their suitability as viable and low-cost alternatives to commercial transwell membranes. 
Overall, this study demonstrates that PLLA microporous nanofilms are good candidates to replace commercial membranes for nanoparticle translocation in vitro studies. Their ability to sustain the adhesion of Caco-2 cells and the formation of a homogeneous monolayer of these cells make PLLA microporous nanofilms suitable for future applications in intestine-mimicking platforms and for more accurate prediction of nanoparticle translocation across the gut barrier. The authors expect that future evolutions of the system will be based on blends of PLLA and other materials (e.g. PDMS and its nanocomposites [45]), which may enable additional features and functionalities.

\section{ACKNOWLEDGMENT}

The authors thank Prof. Arianna Menciassi (The BioRobotics Institute, Scuola Superiore Sant'Anna, Pisa, Italy) for her precious help on result interpretation and discussion and Mr. Carlo Filippeschi for his support in carrying out clean room procedures.

\section{REFERENCES}

[1] S. Sharifi, "Toxicity of nanomaterials," Chem. Soc. Rev., vol. 41, no. 6, pp. 2323-2343, Mar. 2012.

[2] A. Albanese, "The effect of nanoparticle size, shape, and surface chemistry on biological systems," Ann. Rev. Biomed. Eng., vol. 14, pp. 1-16, Apr. 2012.

[3] J.J. Powell, "Origin and fate of dietary nanoparticles and microparticles in the gastrointestinal tract," J. Autoimmunity, vol. 34, no. 3, pp. J226J233, May 2010.

[4] L.W. Peterson, "Intestinal epithelial cells: regulators of barrier function and immune homeostasis," Nature Rev. Immunol., vol. 14, no. 3, pp. 141-153, Mar. 2014.

[5] T. Hartung, "Integrated testing strategies for safety assessment," ALTEX, vol. 30, no. 1, pp. 3-18, 2013.

[6] S. Bhattacharjee, "Surface charge-specif.ic cytotoxicity and cellular uptake of tri-block copolymer nanoparticles," Nanotoxicology, vol. 7, no. 1, pp. 71-84, Apr. 2012.

[7] G.J. Mahler, "Characterization of a gastrointestinal tract microscale cell culture analog used to predict drug toxicity," Biotechnol. Bioeng., vol. 104, no. 1, pp. 193-205, Sep. 2009.

[8] M. Natoli, "Good Caco-2 cell culture practices," Toxicol. in Vitro, vol. 26, no. 8, pp. 1243-1246, Dec. 2012.

[9] L.A. Nkabinde, "Permeation of PLGA nanoparticles across different in vitro models," Curr. Drug Deliv., vol. 9, no. 6, pp. 617-627, Nov. 2012.

[10] A.P. Walczak, "Translocation of differently sized and charged polystyrene nanoparticles in in vitro intestinal cell models of increasing complexity," Nanotoxicology, vol. 9, no. 4, pp. 453-461, May 2015.

[11] H.M. Braakhuis, "Progress and future of in vitro models to study translocation of nanoparticles," Archiv. Toxicology, vol. 89, no. 9, pp. 1469-1495, Sep. 2015.

[12] J. Panchompoo, "Size-effects in the chemical modification of carbon black nanoparticles with 4-nitroaniline," New J. Chem., vol. 34, pp. 2643-2653, Aug. 2010.

[13] P.H.M. Hoet, "Nanoparticles - known and unknown health risks," $J$. Nanobiotechnol., vol. 2, no. 12, Dec. 2004.

[14] S. Takenaka, "Pulmonary and systemic distribution of inhaled ultrafine silver particles in rats," Environ. Health Perspect., vol. 109, suppl. 4, pp. 547-551, Aug. 2001.

[15] Y. Liu, "Understanding the toxicity of carbon nanotubes," vol. 46, no. 3, pp. 702-713, Sep. 2012.

[16] S.S. Teske, "The biomechanisms of metal and metal-oxide nanoparticles' interactions with cells," Int. J. Environm. Res. Public Health, vol. 12, no. 2, pp. 1112-1134, Jan. 2015.

[17] D. Ye, "A TEM protocol for quality assurance of in vitro cellular barrier models and its application to the assessment of nanoparticle transport mechanisms across barriers," Analyst, vol. 140, no. 1, pp. 83-97, Jan. 2015.
[18] A.J. Rosenbloom, "Nanoporous $\mathrm{SiC}$ : A candidate semi-permeable material for biomedical applications," Biomed. Microdev., vol. 6, no. 4, pp. 261-267, Dec. 2004.

[19] H. Wei, "Particle sorting using a porous membrane in a microfluidic device," Lab on a chip, vol. 11, no. 2, pp. 238-245, Jan. 2011.

[20] M.B. Esch, "On chip porous polymer membranes for integration of gastrointestinal tract epithelium with microfluidic "body-on-achip'devices," Biomed. Microdev., vol. 14, no. 5, pp. 895-906, Oct. 2012.

[21] T. Sbrana, "Dual flow bioreactor with ultrathin microporous TEER sensing membrane for evaluation of nanoparticle toxicity," Sens. Act. B: Chem., vol. 223, pp. 440-446, Feb. 2016.

[22] L. Ricotti, "Bioengineering applications of ultra-thin poly(lactic acid) nanofilms towards cell-based smart biomaterials," in: Polylactic Acid: Synthesis, Properties, and Applications. 2012, Nova Science Publishers, New York.

[23] Y. Okamura, "Free-standing biodegradable poly (lactic acid) nanosheet for sealing operations in surgery," Adv. Mater., vol. 21, no. 43, pp. 43884392, Jul. 2009.

[24] V. Pensabene, "Repairing fetal membranes with a self-adhesive ultrathin polymeric film: evaluation in mid-gestational rabbit model," Annals of biomedical engineering, vol. 43, no. 8, pp. 1978-1988, Aug. 2015.

[25] V. Pensabene, "Flexible polymeric ultrathin film for mesenchymal stem cell differentiation," Acta Biomater., vol. 7, no. 7, pp. 2883-2891, Jul. 2011.

[26] L. Vannozzi, "Nanostructured ultra-thin patches for ultrasoundmodulated delivery of anti-restenotic drug," Int. J. Nanomed., vol. 2016, no. 11 , pp. 69-92, Dec. 2015

[27] C.M. Stafford, "A buckling-based metrology for measuring the elastic moduli of polymeric thin films," Nature Mater., vol. 3, no. 8, pp. 545550, Jul. 2004.

[28] N. Ucciferri, "In vitro toxicological screening of nanoparticles on primary human endothelial cells and the role of flow in modulating cell response," Nanotoxicology, vol. 8, no. 6, pp. 697-708, Sep. 2014.

[29] B. Eling, "Biodegradable materials of poly (1-lactic acid): 1. Melt-spun and solution-spun fibres," Polymer, vol. 23, no. 11, pp. 1587-1593, Oct. 1982.

[30] T. Fujie, "Evaluation of substrata effect on cell adhesion properties using freestanding poly (1-lactic acid) nanosheets," Langmuir, vol. 27, no. 21, pp. 13173-13182, Nov. 2011.

[31] S. Giusti, "A novel dual-flow bioreactor simulates increased fluorescein permeability in epithelial tissue barriers," Biotechnology J., vol. 9, no. 9, pp. 1175-1184, Sep. 2014.

[32] G. Ranaldi, "Permeability characteristics of parental and clonal human intestinal Caco-2 cell lines differentiated in serum-supplemented and serum-free media," Toxicol. in Vitro, vol. 17, no. 5, pp. 761-767, Oct. 2003.

[33] H. Bouwmeester, "State of the safety assessment and current use of nanomaterials in food and food production," Trends Food Sci. Technol., vol. 40 , no. 2, pp. 200-210, Dec. 2014.

[34] T. Nakagawa, "Electrical properties of biodegradable polylactic acid film," IEEE Trans. Ind. Appl., vol. 40, no. 4, pp. 1020-1024, Aug. 2004.

[35] D. Garlotta, "A literature review of poly(lactic acid)," J. Pol. Environ., vol. 9, no. 2, pp. 63-84, Apr. 2001.

[36] T. Fujie, "Development of free-standing polymer nanosheets for advanced medical and health-care applications," Polymer J., vol. 48, pp. 773-780, Jul. 2016.

[37] L. Ventrelli, "Nanoscaffolds for guided cardiac repair: the new therapeutic challenge of regenerative medicine," J. Nanomaterials, vol. 2013, ID. 108485, May 2013.

[38] L. Ricotti, "Adhesion and proliferation of skeletal muscle cells on single layer poly (lactic acid) ultra-thin films," Biomed. Microdev., vol. 12, no. 5, pp. 809-819, Oct. 2010.

[39] B. Thierry, "Bioactive coatings of endovascular stents based on polyelectrolyte multilayers," Biomacromolecules, vol. 4, no. 6, pp. 15641571, Nov. 2003.

[40] B. Thierry, "Nanocoatings onto arteries via layer-by-layer deposition: toward the in vivo repair of damaged blood vessels," J. Am. Chem. Soc., vol. 125, no. 25, pp. 7494-7495, May 2003.

[41] A. Folch, "Microengineering of cellular interactions," Ann. Rev. Biomed. Eng., vol. 2, pp. 227-256, Aug. 2000.

[42] J.S. Park, "Quantitative analysis of the combined effect of substrate rigidity and topographic guidance on cell morphology," IEEE Trans. Nanobiosci., vol. 11, no. 1, pp. 28-36, Sep. 2011. 
[43] T. Fujie, "Engineered nanomembranes for directing cellular organization toward flexible biodevices," Nano Letters, vol. 13, no. 7, pp. 3185-3192, Jun. 2013.

[44] M. Natoli, "Good Caco-2 cell culture practices," Toxicol. In Vitro., vol. 26, no. 8, pp. 1243-1246, Dec. 2012.

[45] L..W Jang, "Fabrication of PDMS nanocomposite materials and nanostructures for biomedical nanosystems," IEEE Trans. Nanobiosci., vol. 14 , no. 8 , pp. $841-849$, Dec. 2015

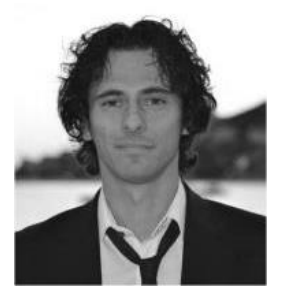

Leonardo Ricotti (IEEE Member from 2009) was born in Volterra (Pisa, Italy), in 1982. He received the B.S. and M.S. degrees in biomedical engineering from the University of Pisa, in 2004 and 2007, respectively, and the Ph.D. degree in biorobotics from Scuola Superiore Sant'Anna (Pisa, Italy), in 2012. From January 2014, he is Assistant Professor with the BioRobotics Institute of Scuola Superiore Sant'Anna (Pontedera, Italy), where he leads the "Micro-Bio-Nano Systems and Targeted Therapies" Lab. He is the author of 60 scientific publications on international journals or conferences and four book chapters and he is the co-inventor of five patents. His research interests include biorobotics, micro/nano-robotics, artificial organs, biomaterials, regenerative medicine, lab-on-chip systems, miniaturized therapeutic devices and nanomedicine. $\mathrm{He}$ is an Associate Editor of the IEEE Transactions on NanoBioscience. He was a recipient of the European Biomaterials and Tissue Engineering Doctoral Award in 2014, the "Massimo Grattarola" Award for the Best Ph.D. Thesis in bioengineering in 2012, and the "LaBS 2005" Award for the best B.S. Thesis in bioengineering in 2005 .

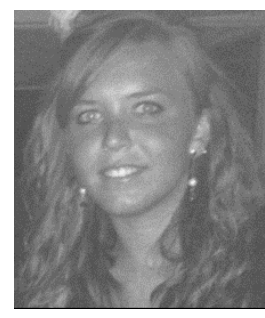

Giulia Gori was born in Pistoia (Italy), in 1988. She received the B.S. and M.S. degrees in biomedical engineering from the University of Pisa, in 2011 and 2014, respectively. She was a Ph.D student in Information Engineering until 2015. She was with the Biorobotics Institute of Scuola Superiore Sant'Anna, Pontedera (Pisa) and with the Research Center "E. Piaggio", University of Pisa (Pisa). Her research interests focus on in-vitro bioinspired models of physiological barriers. In 2014 she was a recipient of the "Silvio Cavalcanti" Award for the best M.S. thesis, entitled "Development of an in-vitro biomimetic device aimed at reproducing the intestinal barrier'. The award motivation highlighted the novelty of the bioengineering methods and technologies used to achieve a biomimetic system aimed at replicating some key features of the human intestinal barrier.

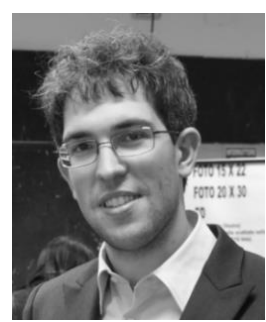

Daniele Cei was born in Pisa (PI) in 1987. He received the B.S. and M.S. degrees in Biomedical Engineering (both with honours) from the University of Pisa in 2009 and in 2011, respectively. He received a Ph.D. in "Automatics, Robotics and Bioengineering", from the University of Pisa in May 2015. His Ph.D. research project was focused on the development and optimization of a dynamic in vitro model as an alternative to animal testing. The project involved both i) computational fluid dynamics modelling and ii) design and realisation of bioreactors to culture and monitor cells under dynamic conditions in order to mimic human pathophysiological environments. Part of this work was performed abroad as a Visiting $\mathrm{PhD}$ Student at the Comprehensive Pulmonary Center of the Helmholtz Zentrum of Munich (Germany), where he gained experience in the physicochemical characterization of aerosol and airborne nanoparticles and in cell cultures techniques. Currently he is part of the R\&D IVTech team, focusing his attention on the design, production and test of peristaltic pumps for in vitro model devices.

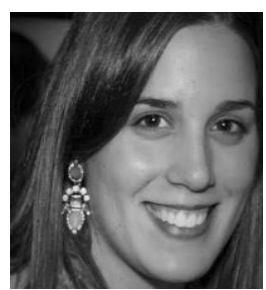

Joana Costa was born in Sever do Vouga (Aveiro, Portugal) in 1991. In 2014 she graduated in bioengineering from the University of Porto (Portugal), with a specialization in Molecular Biotechnology (Integrated Master degree). During this period she was involved in research projects in the fields of Microbiology, Molecular Biology and Tissue Engineering. In 2015 she joined the MICACT European network to work in the integration of electroactive polymers technology for tissue engineering applications. She is currently a Ph.D. student of the University of Pisa and an employee of IVTech, a company focused on the production of systems for the refinement of in vitro models; her research activities take place at the research center 'E. Piaggio' and at the 'Istituto di Fisiologia Clinica' of the 'Consiglio Nazionale delle Ricerche' in Pisa, Italy.

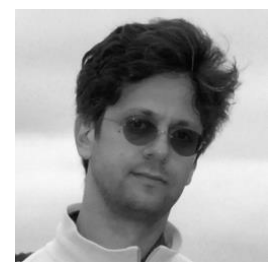

Giovanni Signore was born in Viareggio (Lucca, Italy), in 1978. He received the M.S. degree in industrial chemistry from the University of Pisa in 2003, and the Ph.D. degree in chemical sciences from University of Pisa in 2007. Since 2007 he is post-doc at the NEST laboratory of Scuola Normale Superiore (Pisa, Italy), where he leads the "Synthesis, peptide, an MS Facility" group. He is the author of 37 scientific publications on international journals and he is the co-inventor of two patents. His research interests include synthesis of fluorescent probes, development of engineered nanostructures for targeted delivery and synthesis of biomimetic self-assembled structures.

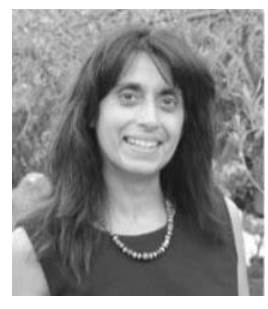

Arti Ahluwalia is Associate Professor of Bioengineering at the University of Pisa, and affiliated with the Research Center "E. Piaggio", where she heads the InVitro Models Group. She is also an associate of the National Council of Research Institute of Clinical Physiology (CNR-IFC), and head of the NanoBioscopy Lab. Her research centres on the interaction between biological systems and man-made devices or structures focused on the creation of organ and system models. The applications span from disease models to in vitro alternatives to animal testing. She is author of over 100 peer- 
reviewed publications and of several inventions which are now commercial products of 2 companies (Kirkstall Ltd, IVTech srl.) she co-founded. 